\title{
Numerical Analysis of the Temperature Distribution of the Rotor
}

\author{
Ran Bo, Guo Zixue, Xie Quanxin, and Zhou Bo
}

\begin{abstract}
In this paper, the finite element method is used to analyze the temperature distribution on the rotor based on the model as the axial symmetrical 2-dimensional steady state. But the effects of the inner radiation and the convection heat transfer in the rotor have not been included. The calculated values are in good agreement with the test values.
\end{abstract}

Index Terms-Finite element method, temperature distribution, centrifuge rotor, numerical analysis.

\section{INTRODUCTION}

Centrifuge is the main equipment for separating isotopes. The movement of the gas in the centrifuge directly affects the separation efficiency of the centrifuge. The temperature of the centrifuge rotor is an important factor to affect the movement of the gas. Because the structure of centrifuge is complex and the rotor is rotating in a sealed system at high speed, the temperature distribution of the entire rotor could not be obtained by test measurement. The heat transfer problems of rotor system involve heat conduction, convection and radiation heat transfer, it is a complex three-dimensional problem. Through simplification and assumption, several simple cases can be simplified to a one-dimensional problem, then get the analytical solutions [1]-[3]. But the accuracy is very bad. In order to get more accurate results, requires a numerical method for solving the heat equation.

\section{PhysicAl Model}

Fig. 1 is a schematic diagram of a typical centrifuge rotor system. Because of the scoops, the rotor system is a non axis symmetric three-dimensional system. Based on the structural characteristics of the centrifuge, the model has been simplified by the following assumptions.

1) Because the rotor is spinning at high-speed, the three-dimensional problem could be simplified to an axis symmetric two-dimensional problem.

2) Ignore the influence of the radiation and convective heat transfer of the inner rotor wall.

3) Ignore the influence of the feed and extraction.

Manuscript received March 1, 2013; revised May 17, 2013.

Ran Bo and Guo Zixue are with the Institute of Physical and Chemical Engineering, Tianjin, China (e-mail: guozx605@139.com, danb03@163.com).

Xie Quanxin is with the Physical Department of Moscow Engineering Physics Institute, State University, Moscow, Russian (e-mail: xxm74@163.com).

Zhou Bo is with the Department of Engineering Physics, Tsinghua Univercity, Beijing, China (e-mail: bozhou.ipce@gmail.com).
4) All parts around the rotor are regarded as the outer boundaries.

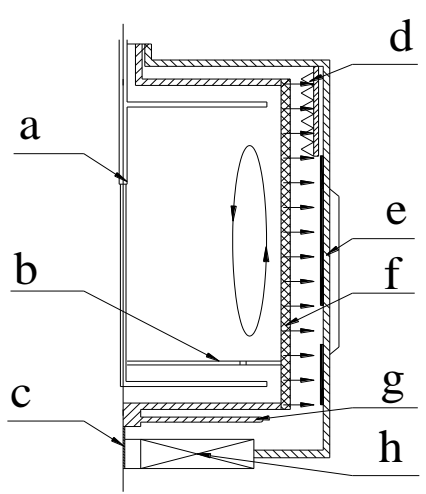

Fig. 1. Schematic diagram of the typical centrifuge rotor system a-feed and extraction device; b-baffle; c-needle bearing; d-molecular pump; e-outer casing; f-rotor; g-motor rotor; h-motor stator.

\section{MATHEMATICAL MODEL}

Based on the physical model as described in the previous section, the temperature equation of the centrifuge rotor [4] is:

$$
\nabla \cdot(k \nabla T)+q=0 \quad \delta(r, z) \in \Omega
$$

Boundary conditions:

$$
\begin{gathered}
\left.T\right|_{\Gamma 1}=T_{01} \\
\left.k \frac{\partial T}{\partial \mathrm{n}}\right|_{\Gamma 2}=0 \\
\left.k \frac{\partial T}{\partial \mathrm{n}}\right|_{\Gamma 3}=q_{0}+\varepsilon \sigma\left(T_{0}^{4}-T^{4}\right)
\end{gathered}
$$

where

$$
\begin{aligned}
& \nabla=\frac{\partial}{\partial r} \vec{e}_{r}+\frac{\partial}{\partial z} \vec{e}_{z} ; \\
& \nabla \cdot=\frac{1}{r} \frac{\partial}{\partial r} r+\frac{\partial}{\partial z} ;
\end{aligned}
$$

$\Omega$ : Solving regional;

$k$ : Thermal conductivity $(W / m \cdot k)$;

$T$ : Temperature of rotor ( $K)$;

$q$ : Intensity of body heat source $\left(W / \mathrm{m}^{3}\right)$;

$T_{01}$ : Temperature of bearing ( $K$ ); 
$\mathrm{n}$ : Outside of the normal direction;

$q_{0}$ : Intensity of surface heat source $\left(W / m^{2}\right)$;

$\varepsilon$ : Combination blackness coefficient between the rotor and outer casing [5];

$\sigma$ : Boltzmann constant;

$T_{0}$ : Ambient temperature $(K)$;

$\Gamma 1$ : Bearing section;

$\Gamma 2$ : Inner surface of rotor;

Г3: Other surface.

Now it's need to solve the equation (1).

\section{EQUATION SOLVING}

\section{A. Galerkin Method}

Introducing a weight function, make the inner product between the left of the (1) and weight function equal to zero:

$$
\int_{\Omega}[\nabla \cdot(k \nabla T)+q] \cdot \omega d \delta=0
$$

where $d \delta$ is the area of infinitesimal.

Using Green formula:

$$
\begin{aligned}
\int_{\Omega} k \nabla T \cdot \nabla \omega d \delta & =\int_{\Omega} q \omega d \delta \\
& +\int_{\Gamma} k \frac{\partial T}{\partial n} \omega^{*} d s
\end{aligned}
$$

Applied the boundary conditions to the (4):

$$
\begin{aligned}
& \int_{\Omega} k \nabla T \cdot \nabla \omega d \delta=\int_{\Omega} q \omega d \delta \\
& +\int_{\Gamma 3} q_{0} \omega^{*} d s+\int_{\Gamma 3} \varepsilon \sigma\left(T_{0}^{4}-T^{4}\right) \omega^{*} d s
\end{aligned}
$$

Equation (4) is nonlinear, it could be solved by iterative methods.

As:

$$
\begin{gathered}
\varepsilon \sigma\left(T_{0}^{4}-T^{4}\right)=\varepsilon \sigma\left(T_{0}^{2}+T^{2}\right)\left(T_{0}+T\right)\left(T_{0}-T\right) \text { Define: } \\
\theta=T^{n+1}-T^{n} \\
E=\varepsilon \sigma\left(T_{0}^{2}+T^{n^{2}}\right)\left(T_{0}+T^{n}\right)
\end{gathered}
$$

where $T^{n}$ is the n-th iteration result, $T^{n+1}$ the iteration result of the $n+1$ time.

Let

$$
\begin{gathered}
\varepsilon \sigma\left(T_{0}^{2}+T^{2}\right)\left(T_{0}+T\right) \approx \varepsilon \sigma T_{0}^{2}+T^{n^{2}}\left(T_{0}+T^{n}\right) \\
T_{0}-T \approx T_{0}-T^{n}+\theta
\end{gathered}
$$

Then

$$
\begin{aligned}
& \varepsilon \sigma\left(T_{0}^{4}-T^{4}\right) \\
& \approx \varepsilon \sigma\left(T_{0}^{2}+T^{n 2}\right)\left(T_{0}+T^{n}\right)\left[T_{0}-\left(T^{n}+\theta\right)\right] \\
& \approx E\left(T_{0}-T^{n}\right)-E \theta
\end{aligned}
$$

Applied this formula to (5):

$$
\begin{aligned}
& \int_{\Omega} k \nabla \theta \cdot \nabla \omega d \delta+\int_{\Gamma 3} E \theta \omega^{*} d s \\
& =\int_{\Omega} q \omega d \delta+\int_{\Gamma 3} q_{0} \omega^{*} d s \\
& +\int_{\Gamma 3} E\left(T_{0}-T^{n}\right) \omega^{*} d s-\int_{\Omega} k \nabla T^{n} \cdot \nabla \omega d \delta
\end{aligned}
$$

The equation (6) could be solved by iterative methods.

\section{B. Finite element Discretization}

Based on the physical model, the solving regional $\Omega$ of the calculation of the temperature distribution should be determined at first. Then it will be divided into the collection of a number of quadrilateral grid cells, as shown Fig. 2. The (6) is suitable for every cell. Using of coordinate transformation, arbitrary quadrilateral cell will be changed into the same parameter cell [6]. The cell's coordinate $(r, z)$ is global coordinate. The coordinate $(\xi, \eta)$ is local coordinate and its range of values is from - 1 to 1 .

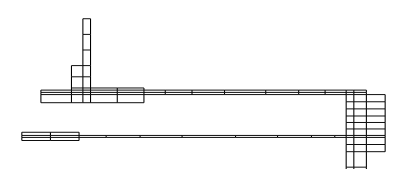

$z$
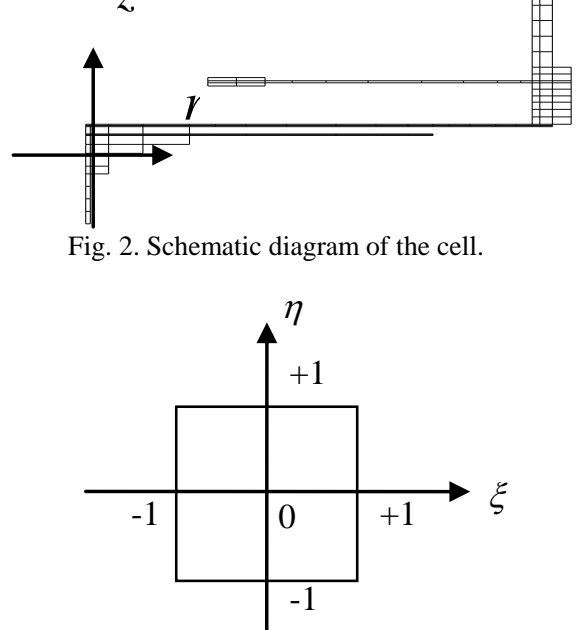

Fig. 3. Schematic diagram of the same parameter cell.

This conversion has the following relationship:

$$
\begin{gathered}
\theta=\varphi^{T} \cdot \theta=\sum_{1}^{4} \varphi_{i} \theta_{i} \\
T^{n}=\varphi^{T} \cdot T^{n}=\sum_{1}^{4} \varphi_{i} T_{i}^{n} \\
r=\varphi^{T} \cdot r=\sum_{1}^{4} \varphi_{i} r_{i} \\
z=\varphi^{T} \cdot z=\sum_{1}^{4} \varphi_{i} z_{i}
\end{gathered}
$$




$$
\omega=\varphi^{T} \cdot \omega=\sum_{1}^{4} \varphi_{i} \omega_{i}=\sum_{1}^{4} \omega_{i} \varphi_{i}=\omega^{T} \cdot \varphi
$$

where

$$
\begin{gathered}
\varphi_{1}=(1+\xi)(1+\eta) / 4 \\
\varphi_{2}=(1-\xi)(1+\eta) / 4 \\
\varphi_{3}=(1-\xi)(1-\eta) / 4 \\
\varphi_{4}=(1+\xi)(1-\eta) / 4
\end{gathered}
$$

For the cell $\Omega_{e}$, the equation could be expressed as:

$$
\begin{aligned}
& \int_{\Omega_{e}} k \nabla \varphi_{i} \cdot \nabla \theta d \delta+\int_{\Gamma 3} E \varphi_{i}^{*} \theta d s \\
& =\int_{\Omega_{e}} q \varphi_{i} d \delta+\int_{\Gamma 3} q_{0} \omega_{i}^{*} d s \\
& +\int_{\Gamma 3} E \varphi_{i}^{*}\left(T_{0}-T^{n}\right) d s-\int_{\Omega_{e}} k \nabla \varphi_{i} \cdot \nabla T^{n} d \delta
\end{aligned}
$$

After simplification, (7) could be expressed as:

$$
\alpha_{i j} \theta_{j}=b_{i}
$$

where $\alpha_{i j}$ is a $4 \times 4$ matrix, named element stiffness matrix; $b_{i}$ is a $4 \times 1$ vector, named as element load vector.

Putting all cells together, a N-dimensional equations:

$$
A \theta=B
$$

where $A$ is a $N \times N$ matrix, named general stiffness matrix; $B$ is a $N \times 1$ vector, named as general load vector. After processing (9) in accordance with the first boundary condition, linear equations will be got. By solving these equations, the temperature of every cell will be found.

\section{Calculated Results And Conclusion}

According the physical model, mathematical model and calculation method which are described in the former paper, a typical example of the temperature distribution of the centrifuge rotor is calculated. The results are shown in Fig. 4.

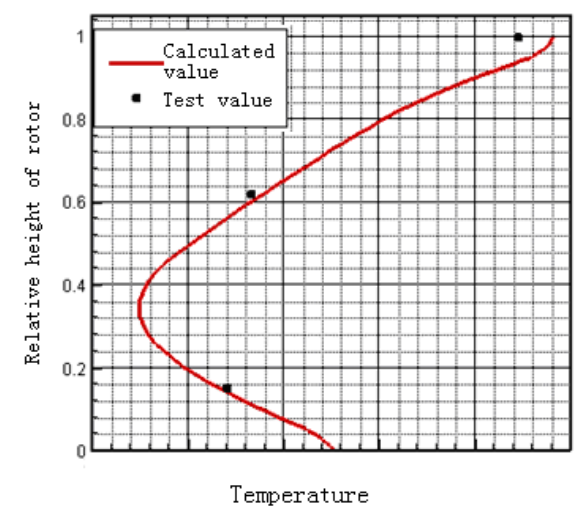

Fig. 4. Typical example of the temperature distribution.
For the figure, the calculated values are in good agreement with the test values. Although the influence of the radiation and convective heat transfer of the inner rotor wall is not considered in this paper, the calculated results could reflect the temperature distribution of the centrifuge rotor. The reason is that the thermal conductivity of the rotor is large and the heat is transported through the rotor. If the material of the rotor is changed and the thermal conductivity becomes smaller, the influence of the radiation and convective heat transfer of the inner rotor wall must be considered.

\section{CONCLUSION}

A conclusion section is not required. Although a conclusion may review the main points of the paper, do not replicate the abstract as the conclusion. A conclusion might elaborate on the importance of the work or suggest applications and extensions.

\section{REFERENCES}

[1] X. W. Song, C. T. Ying, and B. G. Ding, "Anslytical solution of the temperature distribution of the rotor wall in the centrifuge made of two-layers material," J Tsinghua U, vol. 40, no. 10, pp. 5-7, 2000.

[2] Z. J. Huan and B. G. Ding, "The finite element method for temperature distribution of the high speed rotational rotor. journal of engineering thermophysics," vol. 16, no. 4, pp. 466-470, Nov. 1995.

[3] T. KAI, “Analysis of Fluid Flow in Centrifuges," J Nucl Sci Tech, vol. 14, no. 4, pp. 267-281, 1977.

[4] S. M.Yang and W. Q. Tao, Heat transfer, Beijing: Higher Education Press, Aug. 2006.

[5] H. Y. Wang, Handbook of essential formula and data on heat transfer for engineers, New york: Longman Inc, 1977.

[6] X. Q. Kong, Application of finite element method to heat transfer, Beijing: Science Press, 1998 .

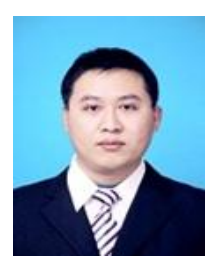

Ran Bo was born in Shehong, Sichuan Province, China PR, on Jan. 4, 1984. He got his master degree from Institute of Physical and Chemical Engineering, Tianjin, China PR, 2010. His major field of study is gas dynamics.

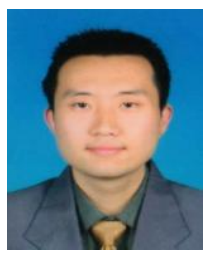

Zhou Bo was born in Changchun, Jilin Province, China PR, on Dec. 27, 1981. He got his bachelor degree from Department of Engineering Physics, Tsinghua Univercity, Beijing, China PR, 2005. His major field of study is Gas dynamics.

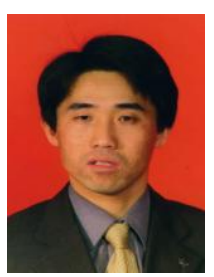

Guo Zixue was born in Bo-Xing, Shandong Provinc, China PR on Dec. 27, 1972. He got his master dgree from Institute of Physical and Chemical Engineering, Tianjin, China PR, 1998. His maijor field of study is Gas dynamics.

Xie Quanxin was born in Hunan province, China PR, on Aug. 26, 1974. He got his Phd degree of maths and physics from physical department of Moscow Engineering Physics Institute, State University, Moscow, Russian, 2007. His major field of study is molecular physics. 